\title{
Factors influencing paediatric nurses' responses to medication administration
}

\author{
Leigh Davis, ${ }^{1}$ Robert S Ware, ${ }^{2,3}$ Damhnat McCann, ${ }^{4}$ Samantha Keogh, ${ }^{5}$ \\ Karen Watson ${ }^{5}$
}

${ }^{1}$ School of Nursing, Queensland University of Technology, Brisbane, Australia

${ }^{2}$ School of Population Health, The University of Queensland, Brisbane, Australia ${ }^{3}$ Queensland Children's Medical Research Institute, Brisbane, Australia

${ }^{4}$ School of Nursing \& Midwifery, University of Tasmania, Launceston, Australia ${ }^{5}$ Brisbane Royal Children's Hospital, Brisbane, Australia

\section{Correspondence to}

Dr Robert S Ware, School of Population Health, The University of Queensland, Herston Road, Herston, OLD 4006, Australia;

r.ware@uq.edu.au

Accepted 6 March 2009 Published Online First 8 April 2010

\section{ABSTRACT}

Objective To evaluate the importance of contextual and policy factors on nurses' judgement about medication administration practice.

Design A questionnaire survey of responses to a number of factorial vignettes in June 2004. These vignettes considered a combination of seven contextual and policy factors that were thought to influence nurses' judgements relating to medication administration.

Participants 185 (67\% of eligible) clinical paediatric nursing staff returned completed questionnaires.

Setting A tertiary paediatric hospital in Brisbane, Australia.

Results Double checking the patient, double checking the drug and checking the legality of the prescription were the three strongest predictors of nurses' actions regarding medication administration.

Conclusions Policy factors, and not contextual factors, drive nurses' judgement in response to hypothetical scenarios.

\section{INTRODUCTION}

Medication errors are a major cause of morbidity and mortality among hospitalised children. ${ }^{1} 2$ Although errors occur at similar rates to adults, they have three times the potential to cause harm in a paediatric setting. ${ }^{3}$ Paediatric patients are especially vulnerable to adverse outcomes from medication error, as they have a reduced capacity for communicating if they are experiencing side effects, immature physiological responses and therefore a limited reserve to withstand a dosing error, ${ }^{12}$ and because of the need for weight-based drug dosing which involve multiple calculations and dilution of stock solutions. ${ }^{4}$

Paediatric nurses are at the end point of the medication administration process, and to date there has been a lack of published data linking causal factors to nurses' clinical judgements and decisionmaking. It has been suggested that one important cause of many reported medication errors could be explained by policy non-adherence or deviation from hospital policy. ${ }^{5}$ Studies have reported routine deviations from, or violation of, policy guidelines by nursing staff and anaesthetists. ${ }^{6}$ The aim of this study is to investigate how contextual and policy factors influence paediatric nurses' judgements relating to medication administration.

\section{METHODS \\ Design}

Paediatric nurses completed a questionnaire survey consisting of six vignettes concerning medication administration issues. Vignettes in the questionnaire were developed to vary according to four contextual and three policy factors, each of which has two levels (in parentheses) that were manipulated within each vignette to examine differences in decision-making. A previous qualitative phase of interviews and focus groups, involving 32 paediatric nurses, informed the development and planning of the questionnaire. ${ }^{7}$ The qualitative phase identified four key contextual factors and three key policy factors thought to influence medication administration practice. The identified contextual factors were the drug's potential harm (harmful/not harmful), the nurse's familiarity with the patient (familiar/not familiar), whether the nurse was working with an experienced colleague (yes/no) and the workload (heavy/light). Although, by their nature, these terms are subjective, we avoided confusion by using extreme examples. For example, when considering whether a drug was potentially harmful, we used commonly prescribed drugs that are perceived to be either not at all harmful, such as paracetamol, or very harmful, such as morphine. The three medication policy factors identified are from the hospital's prescribed medication policy, which all nurses working within the hospital are aware of. They were whether the drug was double checked (yes/no), whether the patient was double checked (yes/no) and whether the prescription was legal (legal order/not legal order). An example of a vignette is displayed in figure 1

For each vignette, nurses were asked how strongly they agreed that: the nurse's actions demonstrated good practice; the medication was safely delivered; the medication was administered according to policy; the average nurse would behave similarly; and their own actions would mirror the actions portrayed in the vignette. Participants rated their level of agreement with each aspect according to a five-point Likert scale ( $1=$ strongly disagree to $5=$ strongly agree). Each nurse was presented with six different vignettes to appraise. Such vignettes are known as factorial vignettes, designed to assess how much influence a specific situational factor has in a given aspect of the contextual or policy factor being investigated.

After the vignettes were developed, a selection of them was assessed for content and face validity by a panel of 10 expert and experienced nurses, none of whom were involved in the questionnaire development. Some of the vignettes were modified according to their comments; the questionnaire was then piloted within a group of eight paediatrics 


Adam is a registered nurse working a day shift on his usual ward in a
paediatric hospital. The ward is quiet and Adam is only caring for one patient.
Beth, a graduate nurse who has been on the ward for 6 weeks, asks him to
check IV acyclovir for a 10 yr old girl that Adam often looks after. Adam
watches while Beth prepares the medication. Although Adam thinks the
medication order is poorly written, Beth thinks she knows what it says. Beth
thanks Adam. Adam says "that's for Jessica in Bed 4" and goes back to his
own work.

Figure 1 Example of a case vignette used in the questionnaire.

nurses outside the sample group who were involved in administering medication. The vignettes were thought to be realistic and representative of a real-life situation.

\section{Study participants}

The study took place in a tertiary paediatric hospital in Brisbane, Australia, between May and July 2004. All clinical paediatric nursing staff $(n=278)$ working in the emergency department, intensive care, medical and surgical wards were invited to participate. Staff working in the operating theatres were excluded due to the selective nature of medication administration in this area.

\section{Data collection}

Covering letters, questionnaires and return-address envelopes were distributed to nursing staff by internal mail. The covering letter explained the purpose, rationale and potential benefits of the proposed study and provided a guarantee of confidentiality and anonymity. Approval for the study was obtained from the hospital's research and ethics committees, as long as no data that could identify an individual were collected.

\section{Statistical analysis}

A hierarchical model was fitted to each of the five questions relating to nurses' judgements about medication administration practice, where the nurses' responses were assumed to be random and the seven contextual and policy factors fixed for each vignette. The clustering on each nurse (because they responded to multiple vignettes) was taken into account. The model estimated the difference in agreement with the statements when the contextual or policy factor was present compared with when it was absent. Thus, the difference measures how much importance nurses placed on the contextual and policy dimensions across the five questions on medication practice. By convention, differences were considered to be of practical value when the absolute difference was greater than 0.4 , and highly significant when greater than 1 . A positive difference denoted agreement with the statement when the factor is present and a negative difference denoted disagreement. All statistical analyses were performed using Stata version 9.1 (Stata Corp., College Station, Texas).

\section{RESULTS}

A total of 185 of 278 (67\%) nurses completed their surveys. Fifty-four per cent of the participants had more than 6 years' experience in paediatric nursing, 46\% were aged 35 years or older, and 74\% were at level NO1. A level NO1 nursing officer is a registered nurse with direct patient care responsibilities but without additional leadership or educational responsibilities.

The relationship between the five questions regarding medication administration and the seven contextual and policy factors is summarised in table 1. Nurses perceived significant positive effects on all five aspects of medication culture practice on each of the three policy factors: if the drug was double checked, if the patient was double checked and if it was a legal prescription. For example, the average score was 0.75 higher (95\% CI 0.63, 0.89) when the drug was double checked than when it was not. In terms of double checking the patient, respondents recorded a highly significant difference when the aspects considered were whether the medication was administered according to policy, and whether the average nurse would behave similarly.

\section{DISCUSSION}

This study found that policy factors, rather than contextual factors, influenced nurses' judgements in response to hypothetical scenarios concerning medication administration. Double checking the patient is the strongest predictor of nurses' actions regarding medication administration. Paediatric nurses appear to recognise and agree upon the fundamental safety aspects built into the current medication policy and recognise that these safeguards should direct decision-making when administering drugs to hospitalised children. Unfortunately, the sensitivity of the topic meant that we were unable to gather enough data about individual respondents to examine whether there were any differences in responses from nurses working in different areas.

Vignettes have historically been used in social science research as a tool to gather information about how clinical

Table 1 Association of the seven contextual and policy factors with each dimension of nursing judgement

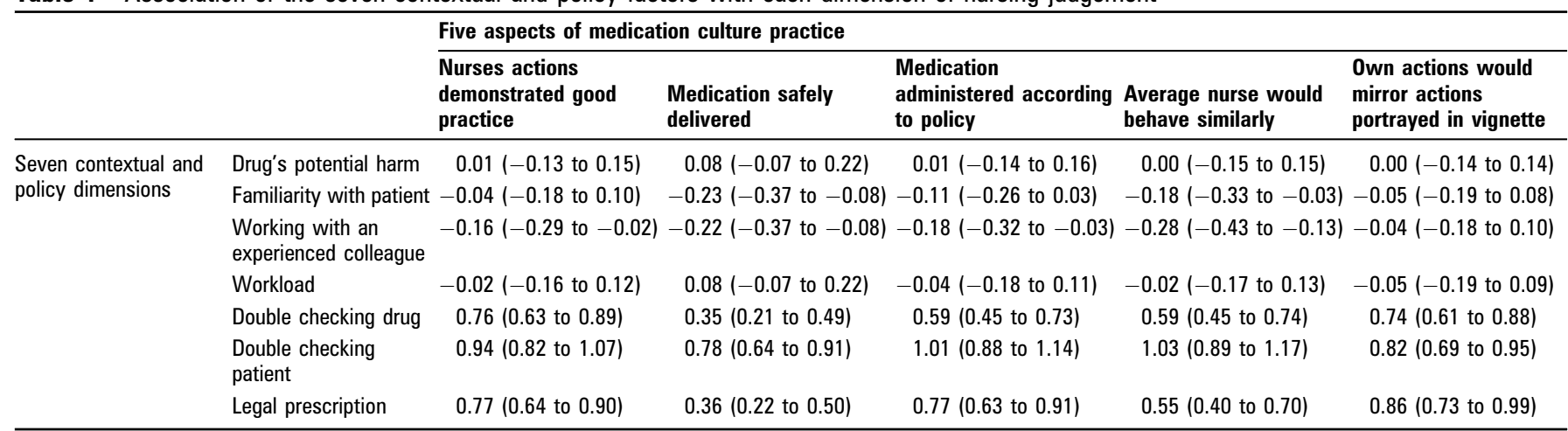

Results are presented as the mean difference $(95 \% \mathrm{Cl})$ of scores when the contextual factor was present compared with when it was absent. A positive difference denotes agreement with the statement when the factor was present. 
judgements are made about patient care situations and the factors that influence those judgements. Vignettes offer a less threatening way to explore sensitive subjects, and their specificity allows contextual influences on judgements to be examined. ${ }^{9}$ We used factorial vignettes in an attempt to capture complex real-life situations, and the conditions of individual choice and judgement, while being able to clearly identify the separate influence that each specific contextual or policy factor has on a nurse's judgement in each aspect being investigated.

Other studies have consistently reported factors occurring in the real-world context which contribute to the potential for error such as stress, fatigue, communication errors, knowledge and skill deficits, ${ }^{10}{ }^{11}$ busy workloads, interruptions and distractions occurring during preparation and administration of medications, ${ }^{11}{ }^{12}$ lack of perceived risk, poor role models and the cultural context which permits unsafe medication practice. $^{6}$

Whether evaluations of hypothetical situations relate to judgements in real life remains an issue. ${ }^{8}$ The questionnaire was carefully constructed to be realistic, with high reliability and internal validity, but it is important to note that any self-report instrument can measure only decision-making and may not predict actual behaviour in similar situations. Our group is conducting further research to determine how closely these findings gleaned from realistic, but hypothetical, vignettes correspond with real-world practice.

\section{Competing interests None.}

Ethics approval Ethics approval was provided by the Brisbane Royal Children's Hospital, Brisbane, Australia.

Provenance and peer review Not commissioned; externally peer reviewed.

\section{REFERENCES}

1. Kaushal R, Bates DW, Landrigan C, et al. Medication errors and adverse drug events in paediatric inpatients. JAMA 2001;285:2114-20.

2. Koren G. Trends of medication errors in hospitalized children. J Clin Pharmacol 2002:42:707-10.

3. Fortescue EB, Kaushal R, Landrigan CP, et al. Prioritizing strategies for preventing medication errors and adverse drug events in paediatric inpatients. Pediatrics 2003:111:722-9.

4. Kozer E, Berkovitch M, Koren G. Medication errors in children. Pediatr Clin North Am 2006;53:1155-68.

5. Armitage G, Knapman $\mathrm{H}$. Adverse events in drug administration: a literature review. J Nurs Manag 2003;11:130-40.

6. Taxis K, Barber N. Causes of intravenous medication errors: an ethnographic study. Qual Saf Health Care 2003;12:343-7.

7. Davis L, Keogh S, Watson K, et al. Dishing the drugs: a qualitative study to explore paediatric nurses' attitudes and practice related to medication administration. Collegian 2005;12:15-20.

8. Ludwick R, Zeller RA. The factorial survey: an experimental method to replicate real world problems. Nurs Res 2001;50:129-33.

9. Lauder W. Factorial survey methods: a valuable but under-utilised research method in nursing research? Nurs Times Res 2002:7:35-45.

10. Leape LL, Bates DW, Cullen DJ, et al. Systems analysis of adverse drug events. JAMA 1995;274:35-43.

11. Osborne J, Blais K, Hayes JS. Nurses' perceptions: when is it a medication error? J Nurs Adm 1999;29:33-8.

12. Mayo AM, Duncan D. Nurse perceptions of medication errors: what we need to know for patient safety. J Nurs Care Oual 2004:19:209-17. 